\title{
MedienPädagogik
}

Zeitschrift für Theorie und Praxis der Medienbildung www.medienpaed.com

Themenheft Nr. 27: Tagungsband: Spannungsfelder und blinde Flecken.

Medienpädagogik zwischen Emanzipationsanspruch und Diskursvermeidung. Herausgegeben von Sven Kommer, Thorsten Junge und Christiane Rust.

\section{Medienpädagogik und digitaler Kapitalismus Für die Stärkung einer gesellschafts- und medienkritischen Perspektive}

Horst Niesyto

\section{Zusammenfassung}

Medienkritik gehörte schon immer zu den zentralen Aufgaben der Medienpädagogik. Die These ist, dass die Medienpädagogik bezüglich einer kritischen Medien- und Gesellschaftsanalyse einen Nachholbedarf hat. Nach einem Blick zurück auf historische Entwicklungslinien der Medienpädagogik wird begründet, weshalb die Stärkung einer gesellschafts- und medienkritischen Perspektive notwendig ist. In einem weiteren Schritt erfolgt eine Annäherung an das Thema ¿Digitaler Kapitalismus〉, indem ausgewählte Beiträge aus verschiedenen wissenschaftlichen Perspektiven skizziert und wichtige Strukturprinzipen herausgearbeitet werden. Der abschliessende Teil thematisiert die gesellschaftliche Verantwortung der Medienpädagogik: Entweder immer mehr zu einem Ausbildungs- und Reparaturbetrieb des digitalen Kapitalismus zu werden (für digitale Technologien und deren (Nebenwirkungen〉) oder sich als kritisch-reflexive Begleitung von medienbezogenen Bildungs- und Lernprozessen zu verstehen.

\section{Media Education and Digital Capitalism}

\begin{abstract}
Media criticism has always been a pivotal task of media education. The assumption is that media education has a need to catch up in regard to a critical analysis of media and society. After looking back at the historical development of media education it is found, why it is necessary to strengthen a socio- and media-critical perspective. In addition, the topic of «digital capitalism is discussed by outlining selected papers from different scientific perspectives and by working out important structural principles of those. The conclusive part addresses the social responsibility of media education: either to become a training and repair company of «digital capitalism» (for digital technologies and their (side effects)) or to understand itself as a critical-reflexive support to mediarelated educational and learning processes.
\end{abstract}




\section{Blick zurück: Von der bewahrpädagogischen Kultur- und Medienkritik zur handlungsorientierten Medienpädagogik}

Jahrzehntelang näherten sich viele Pädagogen/innen den jeweils neuen Medien mit jugendschützerischen Vorbehalten und äusserten primär eine bewahrpädagogische Kultur- und Medienkritik. Die Intention war es, Kinder und Jugendliche vom ‘passiven Medienkonsum` abzubringen und sie vor «verderblichen` Medienwirkungen zu bewahren. Dieter Baacke resümierte hierzu: «Die Nähe des pädagogischen Räsonnements zu einer eher medienabwehrenden Kulturkritik ist daher Tradition und kennzeichnet ein immer wieder gestörtes Verhältnis» (Baacke 1997, 28). Baacke betonte vor allem die mit dieser Form der Kulturkritik verknüpfte normative Kontrollorientierung, die Kinder und Jugendliche letztlich als unmündige Rezipienten behandelt (ebd.).

Die ideologiekritische Medienpädagogik, die sich in den 1960er Jahren im Gefolge der Kritischen Theorie der Frankfurter Schule entwickelte (Horkheimer/Adorno 1944/1960), grenzte sich von der konservativ-normativen Grundausrichtung der bewahrpädagogischen Richtung ab und fokussierte ihre Kritik auf gesellschaftliche Macht- und Herrschaftsverhältnisse. Entsprechende Analysen befassten sich vor allem mit dem Waren- und Tauschcharakter von Kunst- und Kulturgütern und warnten vor der nivellierenden Wirkung einer totalitären Kultur- und Bewusstseinsindustrie (Ehmer 1971). Baacke führte in diesem Zusammenhang aus, dass die ideologiekritische Medienpädagogik theoretische Argumentationsstränge aufnahm, die die Medienpädagogik an sozialwissenschaftliche Debatten anschlussfähig machte und gesellschaftliche Frage- und Problemstellungen aufgriff (Baacke 1997, 47). Die ideologiekritische Medienpädagogik hatte aber auch Schwächen und Grenzen. Diese zeigten sich vor allem in medienzentrierten Analysen zu einem weitgehenden medialen Manipulationsverdacht und "Verblendungszusammenhang», einer elitären Orientierung an ästhetischen Avantgarde-Konzepten und einer Praxisferne bezüglich des Medienerlebens von Kindern und Jugendlichen (vgl. Baacke 1997, 48 f.; Ganguin/Sander 2008, 62 f.).

Der entscheidende Schritt in der Medienpädagogik zur Überwindung medienzentrierter Sichtweisen speiste sich aus sozialisations- und medientheoretischen Überlegungen, die die eigenaktive Leistung der Subjekte bei der Mediennutzung betonen. Dieses Verständnis grenzt sich von monokausalen Medienwirkungstheorien ab (u. a. Kübler 2000, 72 ff.) und korrespondiert mit dem Ansatz in der allgemeinen Sozialisationsforschung, dass Kinder, Jugendliche und Erwachsene die Realität aktiv und produktiv verarbeiten (Hurrelmann 1995). Mediennutzung wird in dieser Perspektive als Teil sozialen Handelns verstanden, es wird nach der Bedeutung der Medien im Alltag und für die Lebensbewältigung gefragt. Bedürfnisund Lebensweltorientierung, Erfahrungsorientierung, Kommunikations- und Projektorientierung entwickelten sich zu Leitkategorien einer handlungsorientierten 
Medienpädagogik (Baacke 1997, 51 ff.; Schorb 1995). Die handlungsorientierte Medienpädagogik verbreitete sich in den 1970er und 1980er Jahren vor allem im ausserschulischen Bereich. Gestützt auf Überlegungen von Benjamin, Brecht, Enzensberger, Negt/Kluge entstanden Konzepte und Projekte aktiver Medienarbeit (vgl. Schell 1993). Im Mittelpunkt steht die Intention, Öffentlichkeit und Erfahrung der Subjekte zusammenzubringen: Medien werden Kindern, Jugendlichen und Erwachsenen in handhabbarer Form zur Verfügung gestellt, damit sie ihre Bedürfnisse und Interessen unabhängig von der Selektions- und Steuerungsmacht der Massenmedien öffentlich artikulieren können.

In Zusammenhang mit gesellschaftlichen Individualisierungs- und Pluralisierungsprozessen sowie der Ausdifferenzierung von Medienangeboten erhielt in den 1980er und 1990er Jahren die Analyse und Förderung medienästhetisch-kultureller Ausdrucksformen ein grösseres Gewicht. Im Mittelpunkt steht eine multiperspektivische Herangehensweise, insbesondere im Kontext der Cultural Studies (u. a. Hipfl/Ernst 2004). Sich mit Medien kreativ und kritisch zu artikulieren wird zum Leitmotiv zahlreicher medienpädagogischer Aktivitäten. Die wahrnehmungsund sozial-ästhetisch orientierte Medienpädagogik betont den Selbstausdruck mit Medien, um Kindern und Jugendlichen im Kontext von Persönlichkeitsbildung und gesellschaftlicher Partizipation symbolische Probe- und Handlungsräume mit Medien zu eröffnen und ihre Medienkompetenzen zu erweitern (u. a. Niesyto 1991; Röll 1998).

In den letzten 15 Jahren stand die Auseinandersetzung mit digitalen Medien, Internetkommunikation, mobilen Medien und digitalen Spielkulturen im Vordergrund medienpädagogischer Aktivitäten. Das exponentielle Wachstum von digitalen Speicherkapazitäten, die Miniaturisierung von Einzelbausteinen, die mobile Einsetzbarkeit und die interaktiven Möglichkeiten in Echtzeitkommunikation sind strukturelle Eigenschaften digitaler Medien, welche einen enormen Schub in der Medienkommunikation ermöglicht haben. In medienpädagogischer Perspektive geht es vor allem darum, wie digitale Medien von Kindern, Jugendlichen und Erwachsenen für reflexive Bildungs- und Lernprozesse, für Kommunikation, Selbstausdruck und gesellschaftliche Partizipation kompetent genutzt werden können. Mit Blick auf die reflexiven Potentiale digitaler Kommunikation und des Social Web betonen verschiedene Autoren/innen u. a. die Kritik-, Ergänzungs- und Kommentierungspraktiken (z. B. bei Weblogs), die Notwendigkeit von Quellenkritik und Kontextualisierung von Informationen (z. B. bei Wikipedia) und einen selbstkontrollierten Umgang mit eigenen Daten sowie die kreativen Möglichkeiten digitaler Medienproduktion, z. B. für das Erzählen von Geschichten (Digital Storytelling). ${ }^{1}$

1 vgl. das Themenheft «Erzählwelten 3.0», Heft 1 (2015) der Zeitschrift medien concret - Magazin für die pädagogische Praxis. 


\section{Stärkung einer gesellschafts- und medienkritischen Perspektive}

Zweifelsohne eröffnen digitale Medien neue Chancen für selbstgesteuerte Bildungs- und Lernprozesse. Dennoch ist darauf hinzuweisen, dass dies kein «Selbstläufers ist - die Nutzung dieser Chancen hängt zu wesentlichen Teilen von den jeweiligen bildungsbezogenen, sozialen und finanziellen Ressourcen ab. Es gibt viele Kinder und Jugendliche, die weder im Rahmen der familiären noch der schulischen Sozialisation hinreichend Anregung und Förderung für einen kompetenten und reflektierten Medienumgang erhalten. Die ICILS-Studie (Bos et al. 2014) und weitere Analysen und Studien der letzten Jahre machten hierauf wiederholt aufmerksam (u. a. Niesyto et al. 2009, Verständig/lske 2014). Gleichzeitig werden problematische Medienentwicklungen immer deutlicher. Zu nennen sind vor allem die enorme Kommerzialisierung im Social Web und der extreme Kontrollverlust hinsichtlich privater Daten. Diese Entwicklungen unterhöhlen systematisch Bildungsanstrengungen und verweisen auf gesamtgesellschaftliche, aber auch auf pädagogische Herausforderungen.

Problematische Medienentwicklungen zu untersuchen war schon immer eine Aufgabe der Medienpädagogik und pädagogischer Medienkritik (Kübler 2006; Niesyto 2008). Der Schritt von der Überwindung bewahrpädagogischer und einseitig ideologiekritischer Ansätze hin zu verschiedenen Formen einer handlungs- und wahrnehmungsorientierten Medienpädagogik bedeutet nicht, auf gesellschaftsund medienkritische Analysen zu verzichten. So verwies z. B. Dieter Baacke - der gerne als einer der Repräsentanten für die subjekt- und kulturtheoretisch orientierte 'Wendes in der Medienpädagogik angeführt wird - immer wieder auf die Notwendigkeit kritischer Analysen zu «kommunikationskulturellen Problemlagen» (Baacke 1997, 76 ff.). Er betonte, dass sich die Medienpädagogik «weder aus der Medienpolitik noch aus dem (Diskurs über Medien〉 der Gesamtgesellschaft zurückziehen» kann, «will sie sich nicht auf pädagogische Provinzen abdrängen lassen, in denen sie nur als (Reparaturbetrieb) funktioniert für das, was ausserhalb von ihr geschieht und zu verantworten wäre» (Baacke 1997, 57).

Mir war es stets ein zentrales Anliegen, die Subjekt- und Handlungsorientierung in der Medienpädagogik mit sozialen und gesellschaftlich strukturellen Fragen zusammenzubringen. So konnte z. B. die Studie über Medienpädagogik und soziokulturelle Unterschiede (Niesyto 2000) eine «Mittelschicht-Lastigkeit» medienpädagogischer Konzepte und die Notwendigkeit einer milieusensiblen Medienkompetenzförderung herausarbeiten. ${ }^{2}$ In den folgenden Jahren gab es weitere Studien über Medien, soziokulturelle Unterschiede und soziale Benachteiligung (u. a. Paus-Hasebrink/Ortner 2007; Wagner 2008). Die Diskussion gewann an Fahrt, als in Zusammenhang mit Untersuchungen zu digital divide und second digital divide

2 In theoretischer Perspektive vgl. auch den Beitrag über «Medien und Wirklichkeitserfahrung - symbolische Formen und soziale Welt» (Niesyto 2002). 
Unterschiede im Zugang und im Gebrauch digitaler Medien immer sichtbarer wurden (u. a. Iske et al. 2007; Niesyto et al. 2009; Theunert 2010; Witzel 2012). Gleichzeitig entstanden Studien, die theoretisch und empirisch der Frage nachgingen, welche Formen des medialen Habitus unter Lehramtsstudierenden zu beobachten sind. Zu nennen sind vor allem die Studien von Ralf Biermann und Sven Kommer (Biermann 2009, Kommer 2010).

Alle diese Studien beförderten zugleich die kritische Hinterfragung von Konzepten zur medialen Selbstsozialisation und Selbstbildung (u. a. Niesyto 2007). So scheint die Überschätzung der Möglichkeiten zu einem medien-autonomen Handeln ein kritischer Punkt in kulturtheoretisch orientierten Kinder- und Jugendmedienstudien zu sein. Konzepte zur Selbstsozialisation mit Medien (u. a. Müller et al. 2004) betonen insbesondere die Eigenleistungen der Individuen im Sinne aktiven, selbstständigen Handelns: Auswahl von Gegenständen, Medienangeboten, Mitgliedschaften, Beziehungsformen und Werthaltungen, Deutungsmustern. Diese Eigenleistungen haben zweifelsohne in Zusammenhang mit dem gesellschaftlichen Prozess der Individualisierung und Globalisierung zugenommen, der von den Individuen in erheblichem Masse mehr eigenverantwortliches Handeln abverlangt. Einerseits verbinden sich damit Chancen zu mehr Selbstständigkeit und Selbststeuerung, andererseits ist jedoch nicht zu übersehen, dass den Menschen unterschiedliche soziale, kulturelle, bildungsbezogene Ressourcen zur souveränen Nutzung von Medien zur Verfügung stehen.

Zugespitzt formuliert: Relevante Teile der Medienpädagogik haben nach der paradigmatischen Wende von der Frage "Was machen die Medien mit den Menschen?» hin zur Frage «Was machen die Menschen mit den Medien?» die Auseinandersetzung mit gesellschaftlichen Veränderungsprozessen subjekttheoretisch verkürzt geführt. Ohne in deterministische Positionen zurückzufallen scheint es notwendig, den strukturellen Einfluss von Medien auf Denk- und Verhaltensweisen von Menschen und die Relevanz unterschiedlicher sozialer, bildungsbezogener und anderer Ressourcen für Bildungsprozesse nicht zu unterschätzen. Mediale Strukturmuster im Kontext gesellschaftlicher Macht- und Herrschaftsverhältnisse herauszuarbeiten und zu reflektieren, ist eine wichtige Aufgabe der pädagogischen Medienkritik. Gleichzeitig stellt sich die Aufgabe, milieusensible Konzepte für die Förderung von Medienbildung und Medienkompetenz (weiter) zu entwickeln, die den unterschiedlichen Medienerfahrungen, lebenslagenbezogenen Ressourcen und soziokulturellen Orientierungen gerecht werden.

Mit der (nachvollziehbaren) Abgrenzung von den Schwächen und Grenzen des ideologiekritischen Ansatzes verband sich leider in der Folgezeit in Teilen der Medienpädagogik eine Theoriebildung und Praxis, die eine kritische Medien- und Gesellschaftsanalyse vernachlässigte. Baacke betonte zwar die zentrale Bedeutung von Medienkritik, konzeptualisierte diese aber primär mit Blick auf 
medienanalytische und ethische Fragen in Verbindung mit «kommunikationskulturellen Problemlagen» (Baacke 1997). Eine systematische und kritische Auseinandersetzung mit gesamtgesellschaftlichen und ökonomischen Fragen und deren Bedeutung für die Medienentwicklung, das Medienhandeln und die Medienbildung erfolgte aber auch bei Dieter Baacke in seinen primär kommunikationskulturell inspirierten Arbeiten nur eingeschränkt.

Bezüglich der theoretisch-konzeptionellen Diskurse zum medienpädagogischen Schlüsselbegriff Medienbildung gelangt Stefan Iske (2014) zu folgender Einschätzung:

Derzeit stellt die Strukturale Medienbildung (Jörissen u. Marotzki 2009) den am weitesten entwickelten theoretischen und empirischen Ansatz der Medienbildung dar. Dies betrifft sowohl die bildungstheoretische wie auch die die medialitätstheoretische Grundlegung. Damit bildet die Strukturale Medienbildung einen zentralen Referenzpunkt des medienpädagogischen Diskurses (Iske 2014, 266).

Zweifelsohne haben die Arbeiten von Benjamin Jörissen und Winfried Marotzki den medienpädagogischen Diskurs der vergangenen Jahre bereichert, wobei an dieser Stelle nicht auf die verschiedenen (und sich auch voneinander unterscheidenden) Beiträge der beiden Autoren weiter eingegangen werden soll. Der theoretische Ansatz der "Strukturalen Medienbildung» - dies sei im Kontext der Thematik des vorliegenden Beitrags betont - bezieht Strukturfragen im Wesentlichen auf die immanente Strukturiertheit der Medien. Es geht um die konstitutiven Aspekte von Medien («Medialität») und um «transgressive Artikulationen im Spannungsfeld von Ästhetik und Medialität» (Jörissen 2015, 55 f.). Analytische Bezüge zu gesellschaftlichen und ökonomischen Strukturfragen, insbesondere im Hinblick auf Macht- und Herrschaftsinteressen, sind (bislang) kein konstitutiver Bestandteil des Ansatzes der «Strukturalen Medienbildung». Auch im Hinblick auf milieusensible Konzepte der Medienbildung gibt dieser Ansatz so gut wie keine Antworten. Die Stärkung einer gesellschafts- und medienkritischen Perspektive ist gerade in einer Situation notwendig, in der Risiken und Problemfelder der Digitalisierung im Kontext ökonomischer und politischer Interessen immer deutlicher werden. Exemplarisch seien folgende Problemfelder genannt: 
- die enorme kommerzielle Verwertung persönlicher Datenprofile und das Entstehen neuartiger Machtstrukturen in Verbindung mit «Big Data» (neue Formen gesellschaftlicher Kontrolle und Steuerung in vielen Bereichen); ${ }^{3}$

- die weitere technische und gesellschaftliche Beschleunigung von Alltags- und Arbeitsabläufen. Stichworte: Tendenz zur Auflösung raumzeitlicher Kontinuitäten und Sozialmilieus, fluide Beziehungen und fragmentarische Identitäten, mediale Aufmerksamkeitserregung, Verlust von Reflexivität und Musse-Zeiten (u. a. Rosa 2005, Niesyto 2012);

- die verstärkte Entwicklung körperlich-digitaler Mensch-Computer-Schnittstellen in Richtung eines homo digitalis (von medialer Extension zu medialer Inkorporation); damit verknüpfte anthropologische, ethische und gesellschaftliche Grundfragen; Stichworte u. a.: Human Enhancement, Bioinformatik (u. a. Damberger 2016).

In letzter Zeit nahmen in der Medienpädagogik die Bestrebungen zu, sich mit Risiken und Problemfeldern der gesellschaftlichen Medienentwicklung intensiver zu befassen. ${ }^{4}$ Es geht dabei nicht darum, Kindern, Jugendlichen und Erwachsenen den Umgang mit digitalen Medien zu «vermiesen` und den «pädagogischen Zeigefingers wieder herauszuholen. Eine handlungsorientierte, kritisch-reflexive Medienpädagogik kann aber nicht darauf verzichten, gesellschaftlich-strukturelle Hintergründe und Zusammenhänge aufzuzeigen und zum Bestandteil von Bildungsprozessen zu machen. Das Thema «Digitaler Kapitalismus» gehört dazu.

Der folgende Teil skizziert Kernpunkte kritischer Analysen zum Thema digitaler Kapitalismus aus verschiedenen wissenschaftlichen Perspektiven, um auf diesem Hintergrund im dann folgenden Teil wichtige «Strukturprinzipien des digitalen Kapitalismus» herauszuarbeiten. Der Beitrag versteht sich als eine (erste) Annäherung an das Phänomen «digitaler Kapitalismus` und ersetzt nicht genauere theoretische

3 «Smartphones merken, wo wir uns aufhalten. Amazon kennt meine Musiksammlung und Google meine Interessen. Der intelligente Stromzähler registriert, wann wir aufstehen, kochen, die Nachttischlampe ausschalten und welches Fernsehprogramm wir gucken. Das neue Internetprotokoll kann jedem Menschen 50 Quadrilliarden Dinge eindeutig zuordnen. Jede Gurke, jeder Fahrschein und jedes Kleidungsstück werden eindeutig identifizierbar. Experten sprechen ehrfürchtig von «Big Data〉 und dem «Internet der Dinge〉. Neurowissenschaftler wollen Gehirn und Computer direkt miteinander verbinden. Unser virtuelles Abbild weiss, wer wir sind, was wir mögen, was wir tun und mit wem wir es tun. Und im Unterschied zu uns selber, vergisst es auch nichts.» (SWR-Podcast Wissen, Reihe «Der vermessene Mensch», Sendung «Das Daten-Ich», 25.07.2014). Zum Phänomen «Filterblase» (Pariser 2012) gibt es inzwischen eine breite Diskussion (u. a. Krüger 2014, Autenrieth 2016); zu Big Data u. a. Gapski (2015).)

4 vgl. z.B. das GMK-Forum 2014 («Doing politics: Politisch agieren in der digitalen Gesellschaft»), die Fachkonferenzen des JFF 2014 und 2015 («Medienpädagogik im digitalen Umbruch»; «Medien, Konsum, Kultur») und in der Sektion Medienpädagogik der DGfE (neben der Aachener Tagung 2015 mit einem Panel zu «Medienpädagogik als Akteur in kapitalistischen, kommerzialisierten und globalisierten Medienwelten») die Theorieforen 2015 und 2016 in Magdeburg zu den Themen «Das umkämpfte Internet» und «Big Data: Implikationen für Medien, Bildung und Gesellschaft». Die Zeitschrift medien+erziehung brachte im August 2014 ein Heft zum Thema «Jugend - Medien - Kommerzialisierung» heraus; die Online-Zeitschrift medienimpulse veröffentlichte im Dezember 2014 mehrere Artikel zum Thema «Steuerung, Kontrolle, Disziplin». 
Analysen und empirische Untersuchungen zu einzelnen Aspekten sowie die Erarbeitung von pädagogischen Konzepten und konkreten Materialien für die medienpädagogische Praxis (Vermittlung und Aneignung von Strukturwissen zum digitalen Kapitalismus etc.). Dies bleibt weiteren Arbeiten vorbehalten.

\section{Digitaler Kapitalismus - Kernpunkte ausgewählter, kritischer Analysen aus verschiedenen wissenschaftlichen Perspektiven}

Beiträge aus wirtschaftswissenschaftlicher und polit-ökonomischer Perspektive Zunächst ist die umfangreiche empirische Arbeit der französischen Sozial- und Wirtschaftswissenschaftler Luc Boltanski und Ėve Ciapello Der neue Geist des Kapitalismus (2003; frz. Erstausgabe 1999) zu nennen. Diese Arbeit, deren Titel auf Max Webers Studie Die protestantische Ethik und der 'Geist〉 des Kapitalismus anspielt, vergleicht frühere mit zeitgenössischer Managementliteratur und arbeitet entlang detaillierter Inhaltsanalysen heraus, wie sich Leitideen des Kapitalismus veränderten und wie der Kapitalismus immer wieder Selbsterneuerungskräfte gerade im ideologischen Bereich entwickelte. Mit Blick auf Sennets Analyse des «flexiblen Menschen» (vgl. Teil "Beiträge aus sozialwissenschaftlicher und kultursoziologischer Perspektive» dieses Beitrags) bietet diese Arbeit Hinweise auf die 〈Verführungskunst〉 des digitalen Kapitalismus: mittels digitaler Technologien ein Maximum an Mobilität und Flexibilität zu erreichen und Netzwerke (soziales Kapital) zu bilden. Allerdings stellt sich die Frage, inwieweit dieser Gesellschafts- und Sozialisationstypus des ständig mobilen und flexiblen «abstract worker» (Böhnisch/Schröer 2001, Kap. II) Menschen überfordert und ausgrenzt, die aus unterschiedlichen Gründen diesem «Anforderungsprofil〉 nicht gewachsen sind bzw. es ablehnen.

Weitere Analysen zur Entwicklung und zur Struktur des Kapitalismus im 21. Jahrhundert liegen u.a. von Thomas Piketty (2014; mit Schwerpunkten zur Dynamik des Kapital-Einkommens-Verhältnisses und zur Struktur der Ungleichheit) und aus marxistischer und polit-ökonomischer Perspektive - von Wolfgang Fritz Haug (2012) über den transnationalen Hightech-Kapitalismus in der grossen Krise (mit den Schwerpunkten Finanzkrise und Hegemoniekrise) sowie von Christian Fuchs (2014) über die internationalen Ausbeutungsformen (Digital Labour and Karl Marx) vor. Die polit-ökonomischen Arbeiten von Haug und Fuchs setzen sich unter Bezug auf «klassische` marxistische Aussagen mit dem Fortbestehen des Gegensatzes von Kapital und Arbeit, damit verbundenen Ausbeutungsformen, Strategien der Kapitalakkumulation und -konzentration bis hin zu imperialen Hegemoniekämpfen auf dem Hintergrund der digital-technologischen Umwälzungen auseinander. 
Aus dem Bereich der Medien- und Internetökonomie sowie der Techniksoziologie gibt es inzwischen diverse Analysen, die z. B. Kapitalkonzentrationsprozesse bei Internetkonzernen detailliert belegen. So machte der Techniksoziologe Ulrich Dolata (2014) von der Universität Stuttgart eine Untersuchung zu Märkte und Macht der Internetkonzerne. Sein Fazit:

Die fünf untersuchten Konzerne prägen nicht nur wesentliche Angebote und Märkte des Internets. Sie regeln als Betreiber der zentralen Infrastrukturen auch die Zugänge zum Netz, strukturieren die Kommunikationsmöglichkeiten der Nutzer und sind wesentliche Treiber des Innovationsprozesses. Nicht Dezentralisierung, Demokratisierung und Kooperation, sondern Konzentration, Kontrolle und Macht sind, so die These, die Schlüsselprozesse und -kategorien, mit denen sich die wesentlichen Entwicklungstendenzen des (kommerziellen) Internets angemessen erfassen lassen (ebd., Abstract). ${ }^{5}$ Unter dem Aspekt einer möglichen Transformation kapitalistischer Wirtschaftsweisen sind u. a. die Analysen des US-amerikanischen Wissenschaftlers Jeremy Rifkin zu nennen, der sich in den letzten Jahren insbesondere mit neuen Formen der Organisation des Lebens und des Wirtschaftens durch die gemeinsame Nutzung digitaler Technologien auseinander setzte (Rifkin 2014). In Abgrenzung zu Formen von «digitalen Commons» und «share economy», die aufgrund problematischer Arbeitsverhältnisse und anderer Faktoren einer heftigen Kritik ausgesetzt sind (vgl. das Beispiel des Taxiunternehmens Uber), geht es Rifkin und anderen um Formen eines nicht-kapitalistischen Wirtschaftens, welches auf peer-to-peer-Netzwerken, kollaborativen Commons, universellem Zugang und Offenheit für alle basiert (vgl. auch Habermann 2015). Rifkin prognostiziert durch die Verbreitung von kollaborativen Commons eine erhebliche Schwächung kapitalistisch strukturierter Systeme (kritische Anmerkungen zu Rifkins Ansatz vgl. Moser 2016, 216 f.). Die Auseinandersetzung mit alternativen ökonomischen Konzepten (und damit verknüpften Vorstellungen von gesellschaftlichem Zusammenleben) ist wichtig, weil es nicht nur um die (notwendige) Kritik an kapitalistisch strukturierten Wirtschaftsweisen, sondern auch um Überlegungen für alternative Formen des Wirtschaftens unter Nutzung digitaler Technologien geht.

\section{Beiträge aus sozialwissenschaftlicher und kultursoziologischer Perspektive}

Der US-amerikanische (Kultur-)Soziologe Richard Sennett wurde im deutschsprachigen Raum vor allem mit seinem Buch Der flexible Mensch. Die Kultur des neuen Kapitalismus (1998) bekannt. Sennett arbeitet heraus, dass die kapitalistische Ökonomie auf das Kurzfristige ausgerichtet ist und hierfür in extremer Weise eine

5 Bei den fünf untersuchten Konzernen handelt es sich um Google, Facebook, Apple, Amazon und Microsoft. 
flexible Spezialisierung benötigt. Der flexible Mensch, der sich mit dem globalisierten Netzwerkkapitalismus arrangiert und sich permanent fit hält für die Anpassung an neue Marktentwicklungen, bindet sich - so Sennett - nicht zu sehr an Zeit und Ort und nimmt Fragmentierungen in den lebensweltlichen Zusammenhängen hin bzw. betrachtet sie als einen Gewinn. «Postmoderne Auffassungen des Ich [...] betonen Bruch und Konflikt, aber nicht die Kommunikation zwischen den fragmentierten Teilen des Ich» (ebd., 198).

An dieser Problemlage setzt der Beitrag der Soziologen und Sozialpädagogen Lothar Böhnisch und Wolfgang Schröer (2001) an. Die Autoren analysieren ökonomische Modernisierungsprozesse als «digitalen Kapitalismus» und diagnostizieren eine «digitale Anomie» (ebd., 154 ff.). In einer Welt der grenzenlosen Flexibilisierung und Simulation wird der Mensch zu einem Abstraktum gemacht, dem es immer schwerer fällt, angesichts des Externalisierungszwangs («abstract worker») eigene Selbst- und Weltentwürfe zu entwickeln. Das digitale Prinzip strukturiert die Wirtschafts- und Arbeitsgesellschaft im globalen Raum und im Programm der Digitalisierung trifft sich die Logik der neuen Medien mit der Logik der kapitalistischen Ökonomie. In einer späteren Veröffentlichung wird in Verbindung mit dem digitalen Kapitalismus ein Sozialisationstyp beschrieben:

Gelebt wird mehr und mehr von Situation zu Situation, von Punkt zu Punkt [...] So hat sich eine mediengesellschaftliche Sozialisationsweise entwickelt, die dem digitalen Prinzip gehorcht und einen entsprechenden Sozialisationstyp durchzusetzen versucht, der vor lauter Punkten das Ganze nicht mehr sieht. Die oszillierenden Bildpunkte lenken von den Machtstrukturen ab» (Böhnisch et al. 2009, 133).

Wenngleich die Einschätzung, dass die Sozialisationsweise dem digitalen Prinzip "gehorcht», sehr medienzentriert formuliert ist, so machen die Autoren auf den Zusammenhang von technologisch-wirtschaftlichen Strukturprinzipien und mediengesellschaftlicher Sozialisationsweise aufmerksam - ein Zusammenhang, der auch für die medienpädagogische Theoriebildung und Forschung relevant ist.

Die Punkt-zu-Punkt-Struktur und damit verknüpfte Fragen der Wahrnehmung und Erfahrung von Wirklichkeit sind auch für den Soziologen Hartmut Rosa unter der Perspektive veränderter Zeitstrukturen ein zentrales Thema. Im Jahre 2005 veröffentlichte er eine Abhandlung zum Thema Beschleunigung. Die Veränderung der Zeitstrukturen in der Moderne. In seinem theoretischen Grundgerüst unterscheidet Rosa drei zentrale Dimensionen sozialer Beschleunigung:

- die technische Beschleunigung, die Rosa vor allem auf die schnellere Produktion und Distribution von Gütern und Informationen bezieht. Er verweist u. a. auf die digitalen Technologien, die Informationen in Lichtgeschwindigkeit, in Echtzeit weltweit zugänglich zu machen; 
- die Beschleunigung des sozialen Wandels. Darunter versteht Rosa das Tempo, mit dem sich Praxisformen, Handlungsorientierungen und Beziehungsmuster verändern. Er spricht von einer "Gegenwartsschrumpfung» und einer "Steigerung der Verfallsraten von handlungsorientierenden Erfahrungen und Erwartungen» (Rosa 2005, 133);

- die Beschleunigung des Lebenstempos. Hierzu zählt Rosa Phänomene wie die Verkürzung oder Verdichtung von Handlungsepisoden pro Zeiteinheit, die Zunahme von Multitasking und damit verbundenen Empfindungen der Zeitnot, des Zeitdrucks, eines stressförmigen Beschleunigungszwangs und der Angst, nicht mehr mitzukommen (ebd., $135 \mathrm{ff}$.).

Rosa analysiert, dass die zunehmenden Kurz-Kurz-Muster der Zeitwahrnehmung dazu führen, dass Erlebnisse episodisch bleiben und nicht mehr miteinander, mit der Geschichte und der je eigenen Identität verknüpft werden. Im Ergebnis beginne die Zeit gewissermassen an beiden Enden zu rasen: «Während der als kurzweilig (und oft als stresshaft) empfundenen Aktivitäten vergeht sie sehr rasch, doch zugleich scheint sie im Rückblick zu «schrumpfen», weil sie kaum Erinnerungsspuren hinterlässt.» «Die durch das Kurz-Kurz-Muster gekennzeichnete Gesellschaft» - so sein Fazit - «könnte sich daher als eine gleichermassen erlebnisreiche wie erfahrungsarme Gesellschaft erweisen» (ebd., 470).

Neben den Beschleunigungsprozessen sind es die quantitativen Wachstumsprozesse, die Rosa bereits 2005 in den Mittelpunkt seiner Analysen rückte. «Beschleunigung zum Zwecke der Steigerung» - dies sei eine Handlungslogik des Systems der kapitalistischen Wirtschaft, «in dem Beschleunigung zu einem unentrinnbaren, in die materialen Strukturen der Gesellschaft eingelassenen Sachzwang wird» (ebd., 257 f.: «Zeit ist Geld: Der ökonomische Motor»).

In dem 2009 gemeinsam mit Klaus Dörre und Stephan Lessenich herausgegebenen Band Soziologie - Kapitalismus - Kritik. Eine Debatte führte Hartmut Rosa diese Argumentation fort. Er betont zunächst, dass eine zeitdiagnostisch fundierte Gesellschaftskritik zum Kerngeschäft der Soziologie gehört und jede Gesellschaftskritik der Gegenwart notwendig auch Kapitalismuskritik sein muss. Sein Kernargument: «Wenn wir an den für die moderne Marktwirtschaft und die moderne Demokratie grundlegenden Massstäben der Autonomie (und der Authentizität) festhalten wollen, dann verursacht das kapitalistische Wirtschafts- und Beschleunigungsregime schwerwiegende Pathologien wachsenden Ausmasses» (Rosa 2009, 93). Im Folgenden entfaltet er dieses Argument und analysiert Wachstum und Beschleunigung als prozessleitende Prinzipien der Kapitalakkumulation (ebd.: 98 ff.), wobei - so Rosa - Wirtschaftswachstum und soziale Beschleunigung aufs Engste verzahnt sind. Rosa spricht von einem «Beschleunigungstotalitarismus» (ebd., 117 f.) mit den Folgen einer «Kolonialisierung von Lebenswelten» (Habermas 1987) und «Entfremdungserfahrungen als Störung der «Weltbeziehungen` der Subjekte» 
(ebd., 120). Mit diesen Analysen und Begrifflichkeiten knüpft er auch an Beiträge der «Kritischen Theorie» an (vor allem an Horkheimer und Adorno, teilweise auch an Habermas), insbesondere zur Kritik an einer kapitalistisch strukturierten Ökonomie und damit verknüpften Enteignungs- und Entfremdungsprozessen in Lebenswelten.

Aus sozialwissenschaftlicher Perspektive sind auch die Beiträge von Jörn Lamla hervorzuheben. Er befasste sich mit Konsumpraktiken in der virtuellen Alltagsökonomie und Fragen der Verbraucherdemokratie und setzte sich in diesem Zusammenhang mit dem «kulturellen Kapitalismus im Web 2.0» auseinander (Lamla 2010). Gegenstand seiner Analyse sind - in Anlehnung an die Theorie sozialer Welten von Anselm Strauss - die «Prozesse der Segmentation, Intersektion und Aushandlung kultureller und ökonomischer Praktiken» (ebd., 11). Im Mittelpunkt seines Erkenntnisinteresses stehen Fragen danach, wie sich die strukturellen Widersprüche des kulturellen Kapitalismus im Internet manifestieren, wie sich öffentliche und kommerzielle Räume überlagern und wie sich neue, posttraditionale Gemeinschaften um digitale Marken herausbilden. Zur Beantwortung dieser Fragen stellt Lamla vier Modelle bzw. Thesen zur Relation von kulturell bzw. ökonomisch ausgerichteten sozialen Welten vor:

a) die Homologiethese, die davon ausgeht, "dass die strukturellen Spannungen zwischen den kulturellen und den ökonomischen Praktiken, Austauschbeziehungen und Innovationsprozessen in Grenzen gehalten werden, weil auf einer tieferliegenden Ebene eine Wahlverwandtschaft zwischen den beiden Seiten besteht oder sich neu herausbildet» (ebd., 20);

b) die Fragmentierungsthese (ebd., 21), die die Dominanz des kapitalistischen Regimes der flexiblen Akkumulation (und damit verbundene Ausbeutungsund Verwertungsinteressen) betont und analysiert, wie ökonomische Verwertungsinteressen Kultur und andere gesellschaftliche Bereiche unterwerfen und beständig auf der Suche nach neuen Märkten sind (Formen des Übergriffs, der Kolonialisierung von Lebenswelten, vgl. Habermas 1987);

c) die Kopplungsthese (ebd.), die aufzeigt, wie sich Kultur und Ökonomie im kulturellen Kapitalismus gegenseitig so durchdringen, dass beide Bereiche daraus Nutzen ziehen und sich weiter ausdifferenzieren (funktionale Differenzierungstheorie);

d) die Aushandlungsthese, die die Notwendigkeit von Aushandlungsprozessen zwischen Kultur und kapitalistischer Ökonomie betont, um Konflikte zu lösen bzw. nach Kompromissen zu suchen (z. B. durch «Stakeholder-Dialoge», ebd. 22).

Lamla diskutiert diese Thesen unter Bezug auf vier ausgewählte Phänomenbereiche im Web 2.0 (Social-Network, Prosumtion, soziale Produktion und Tauschbörsen). Er 
resümiert, dass im Vordergrund Spannungen und Konflikte stehen, die sich zwischen ökonomischen und sozialen Welten im kulturellen Kapitalismus ergeben:

Dass diese Welten im Web 2.0 verstärkt aufeinandertreffen und wechselwirken, ist nicht nur aufgrund der Wertschöpfungsprobleme des Kapitalismus im Zeitalter der Wissensökonomie hoch plausibel, sondern konnte in allen vier Untersuchungsfeldern auch nachgewiesen werden. Welche evolutionäre Dynamik damit einhergeht, bleibt hier allerdings offen und erfordert weitere Forschung (ebd., 29f.).

Lamla betont damit zum einen die komplementäre Funktion der vier Theoriemodelle für das Verständnis der analysierten Prozessdynamiken, äussert aber mit Blick auf sich abzeichnende Dominanzen die Vermutung, dass der individualistische Habitus, kulturelle Fragmentierungstendenzen und neue Kopplungsformen eine Allianz bilden, die Aushandlungsprozesse im digitalen Kommunikationsraum eher marginalisieren werden. Die Analysen von Lamla sind wichtig, weil sie die Wechselwirkung von ökonomischen Strukturen und kulturellen Praxisformen zum Gegenstand haben und anschlussfähig für Fragen sind, wie unter diesen Bedingungen Kinder, Jugendliche und Erwachsene ihr Medienhandeln entwickeln und wie sie unterstützt werden können, Zusammenhänge zwischen wirtschaftlichen Interessen und eigenem Medienhandeln zu erkennen. ${ }^{6}$

\section{Beiträge aus kommunikations- und medienwissenschaftlicher Perspektive}

Der US-amerikanische Wissenschaftler Dan Schiller veröffentlichte bereits 1999 ein Buch über Digital Capitalism (Schiller 1999). Seine Analysen beziehen sich vor allem auf die Entwicklung transnationaler Telekommunikationsunternehmen und auf die Geschichte des Internet:

In this book, I show that the Internet and, indeed, the greater telecommunications system with which the Internet has intertwined comprise a leading edge of this epic transnationalization of economic activity (...) Networks are directly generalizing the social and cultural range of the capitalist economy as never before. That is why I refer to this new epoch as one of digital capitalism (Schiller 1999, XIV).

Schiller arbeitet heraus, wie sich ein Transformationsprozess durch Kapitalbildungsprozesse zu einem digitalen Kapitalismus vollzieht und spricht auch negative Auswirkungen auf das Bildungs- und Sozialwesen an: «digital capitalism has already begun to prey on education, placing some of the most sensitive processes of social learning at the mercy of a proprietary market logic» (ebd.).

6 vgl. in diesem Zusammenhang den Beitrag von Jörn Lamla auf der 11. Interdisziplinären Tagung in München (JFF und BLM): http://www.id-tagung.de/2015/12/22/erstes-video-online-prof-lamlaueber-digtales-leben-im-kulturellen-kapitalismus/ 
In demselben Jahr wie Dan Schiller publizierte Peter Glotz ein Buch über Die beschleunigte Gesellschaft. Kulturkämpfe im digitalen Kapitalismus (Glotz 1999). Glotz war Politiker (SPD) und Kommunikationswissenschaftler. Er analysierte vier Basistrends, die mit der Digitalisierung eng verbunden sind: Dematerialisierung, Dezentralisierung, Beschleunigung, Globalisierung. Glotz verwies auf die «Punkt-zu-Punkt-Struktur» neuer, digitaler Kommunikationsformen und analysierte die Verstärkung eines nomadischen Lebensstils. Seine Prognose zur weiteren Gesellschaftsentwicklung ist auf dem Klappentext des Buchs zusammengefasst: «Ungebremst und ungesteuert führt die durch die Digitalisierung angestossene Entwicklung unweigerlich zu einer Spaltung der Gesellschaft: in eine Elite, die das hohe (Arbeits-)Tempo bereitwillig mitmacht, und in eine neue Unterschicht, die sich zu einem guten Teil aus Aussteigern und Verweigerern speist» (ebd.). Glotz prognostizierte eine Zweidrittel-Gesellschaft (konfliktfähige Gruppen werden mitgenommen, konfliktbeladene ausgegrenzt) und neue Kulturkämpfe zwischen Beschleunigung und Entschleunigung - diese Prognose enthält durchaus Elemente aktueller Entwicklungen. Allerdings ist seine Analyse insofern affirmativ, als sie die Entwicklung als gegeben hinnimmt und keine bildungs- und gesellschaftspolitischen Alternativen entwickelt.

Die weltweite Finanzkrise 2008/2009 lenkte den Blick verstärkt auf die Ursachen und Konsequenzen finanzkapitalistischer Strategien und neoliberaler Politik. Es erschien eine Vielzahl von Essays und Abhandlungen unterschiedlicher Qualität, die sich mit diesen Phänomenen auseinandersetzten, z. B. Frank Schirrmachers Buch Payback (2009), Yvonne Hofstetters Sie wissen alles (2014) oder Joseph Vogls Das Gespenst des Kapitals (2011). Die Abhandlung von Vogl legt den Fokus auf die Analyse des Finanzkapitals. Es ist beeindruckend, wie ein Literatur- und Medienwissenschaftler, der an der Humboldt-Universität zu Berlin einen Schwerpunkt im Bereich Medien hat, aus einer Aussenperspektive eine Analyse des finanzkapitalistischen Systems bietet.

Dabei geht es allerdings nicht um Rezepte für den nötigen Umbau des gegenwärtigen Wirtschaftssystems. Es handelt sich vielmehr um den Versuch zu verstehen, wie die moderne Finanzökonomie eine Welt zu verstehen versucht, die durch sie selbst hervorgebracht wurde. Das «Gespenst des Kapitals' erscheint darin als Chiffre für jene Kräfte, von denen unsere Gegenwart ihre Gesetze empfängt. (ebd., 8)

Vogl setzt sich kritisch mit ökonomischen Modellen (besser gesagt: Ideologien und Legenden) vom «Gleichgewicht des Marktes» auseinander, analysiert das Zusammenspiel von Terminhandel, neuen Berechnungsverfahren und Informationstechnologien und arbeitet die historische Transformation heraus (von Währungsstandards jeglicher Art zu einem Informationsstandard als Basis der Weltfinanz): 
Sofern Preise auf den Finanzmärkten zugleich Informationen über die Zukunft von Preisen kompilieren, sind im Zahlungsverkehr Informationen über Geld wichtiger als Geld selbst geworden (...) Dies reicht bis zum gegenwärtigen Wettlauf im high frequency trading, in dem neueste Technologien einen Vorteil von Millisekunden bei der Abfrage von Marktinformationen schaffen. (ebd., 108).

Vogl betont, dass der Kapitalismus kein homogenes System ist. Zentral ist aber der Mechanismus der Kapitalreproduktion: «Von einer kapitalistischen Ökonomie soll dort gesprochen werden, wo die «künstliche` oder chrematistische Reproduktion von Kapitalformen samt ihren Dynamiken und Krisen zum Mass gesellschaftlicher Vitalität geworden ist» (ebd., 131). Ähnlich wie Sennett und andere Soziologen analysiert Vogl Zusammenhänge zwischen den Mechanismen der Kapitalreproduktion und marktförmigen Veränderungen im Alltagsleben der Menschen und gesellschaftlichen Institutionen: «Genetische Ausstattung, Erziehung, Bildung, Wissen, Gesundheit und Familienplanung werden gleichermassen dem ‘ökonomischen Ansatz» unterworfen (ebd., 138) - «das Leben des Gesellschaftskörpers [wird] mit der Bewegung des Kapitals koordiniert» (ebd., 140).

Aus medien- und kommunikationswissenschaftlicher Perspektive leistete in den vergangenen Jahren vor allem Friedrich Krotz im Rahmen einer Mediatisierungsforschung theoretische und empirische Beiträge zur Frage «nach dem sozialen und kulturellen Wandel auf Mikro-, Meso- und Makroebene im Kontext des Wandels der Medien und umgekehrt» (Krotz 2014, 8). Auch Krotz spricht von einer «Kolonialisierung von Lebenswelten» und einer Entwertung von menschlichen Erfahrungen zugunsten von Datensammlungen auf dem Hintergrund von Profitbestrebungen ( KKooperation kommerzieller Interessen und medialer Kontrolle»; Krotz 2012, 14f.). Mit «Kommerzialisierung» im Kontext der Internet-Entwicklung bezeichnet er renditeorientierte Geschäftsmodelle, deren Hauptziel es ist, durch die systematische Nutzung und Auswertung von sehr vielen Nutzerdaten Geld zu verdienen und Märkte zu erobern (ebd., 10):

[...] die Entstehung neuer Unternehmen mit eigenständigen Geschäftsmodellen im Netz als auch die zunehmende Einwanderung ralter' Unternehmen dorthin machen Mediatisierung und Kommerzialisierung derzeit zu einem miteinander verwobenen Geschehen [...] Das Internet wird somit einerseits zum zentralen Nervensystem für die gesamte Ökonomie, das Gleiche lässt sich über die meisten anderen gesellschaftlichen Bereiche und damit auch über Politik und Gesellschaft insgesamt sagen, wobei langfristigen Folgen dieser Entwicklungen in keiner Weise klar sind. (ebd.)

Die neue «Punkt-zu-Punkt-Erreichbarkeit» ist für Krotz einer der zentralen Gründe, weshalb das Internet sowohl für Ökonomie und andere gesellschaftliche Bereiche als auch für die Nutzer/innen wichtig ist (ebd., 11). Hier trifft sich seine Analyse mit 
der von Glotz (1999) und Böhnisch et al. (2009). Im «Kampf ums Internet» (ebd., 9) sieht Krotz derzeit nicht zivilgesellschaftliche und partizipative Interessen dominieren: «Die Richtung der Entwicklung wird derzeit weitgehend von der Technikentwicklung und den ökonomischen Interessen bestimmt» (Krotz 2015, 10). Dieses Fazit deckt sich mit den zuvor erwähnten Analysen zu Kapitalakkumulationsprozessen in der Internet-Industrie und mit Untersuchungen (zumindest in Teilen) zu mediatisierten Geschäftsmodellen wie sie von Pfadenhauer und Grenz (2012) vorgelegt wurden. Kritisch ist anzumerken, dass Krotz in seinen Beiträgen zwar von «ökonomischen Interessen» und "Profitbestrebungen» spricht; ${ }^{7}$ eine genauere Auseinandersetzung mit den ökonomischen Strukturprinzipien unterbleibt jedoch. ${ }^{8}$

\section{Strukturprinzipien des digitalen Kapitalismus}

Der von Karl Marx im 19. Jahrhundert analysierte Kapitalismus (Marx 1867) hat sich in vielerlei Hinsicht weiterentwickelt. An dieser Stelle können nicht die Geschichte und die Theorien zum Kapitalismus und seinen verschiedenen Varianten dargestellt und diskutiert werden. ${ }^{9}$ Auch kann es nicht darum gehen, sich pauschal auf ein ‘Orientierungssystem` zu beziehen, welches der Komplexität und Vielschichtigkeit gesellschaftlicher Verhältnisse und Lebensweisen nicht gerecht werden würde. Darauf haben u. a. diverse Denkansätze der Cultural Studies in unterschiedlicher Akzentuierung strukturalistischer und kulturalistischer Ansätze hingewiesen (Göttlich 2015; Thomas 2015). Hierzu gehört auch ein differenzierter Blick auf die wechselseitigen Beziehungen von 〈Basis〉 und ¿Überbau〉, die Überwindung eines Denkens in Antagonismen und in Kategorien von «richtigem» und ‘falschem» Bewusstsein resp. Bedürfnissen. Gleichwohl bleibt die Frage nach der Kritik bestimmter Strukturmuster und die Frage nach Transformationsmöglichkeiten hin zu einer Wirtschafts- und Gesellschaftsentwicklung, die - auch in unseren Breitengraden - einer wachsenden Kluft zwischen Arm und Reich und einem deutlichen Trend zur dauerhaften Bildungsbenachteiligung eines relevanten Teils der hiesigen Gesellschaft entgegenwirkt.

7 Ökonomische Interessen und kapitalistisches Profitstreben sind nicht dasselbe. Es gibt auf der Welt unterschiedliche ökonomische Konzepte und Handlungslogiken. Die kapitalistische Ökonomie ist eine davon (wobei es davon verschiedene Varianten gibt). Auch «kommerzielle Interessen» sind nicht dasselbe wie kapitalistisches Profitstreben. «Kommerz» bezeichnete früher «Handel» (vgl. die lateinische Wortwurzel). Erst unter hoch entwickelten kapitalistischen Produktionsverhältnissen wurde «Kommerzialisierung» zur Bezeichnung für einen Prozess der Übertragung kapitalistischer Handlungslogiken (insbesondere maximales Profitstreben) auf andere gesellschaftliche Bereiche.

8 Vgl. in diesem Zusammenhang auch die Anmerkung von Kübler (2015) in einer Rezension zu dem von Friedrich Krotz et al. herausgegebenen Handbuch «Cultural Studies und Medienanalyse», dass in diesem Handbuch ökonomische Fragestellungen eher zurückhaltend thematisiert werden.

9 «Schon 1918 wurden in der damaligen Wirtschaftsliteratur 111 verschiedene Definitionen von Kapitalismus gefunden» (Herrmann 2015, 3). 
Es ist interessant, dass spätestens mit der Finanzkrise 2008/2009 kapitalistische Strukturen und ihre Auswirkungen auf nahezu alle gesellschaftlichen Bereiche auch in wissenschaftlichen Kontexten wieder vermehrt zum Gegenstand von Analysen geworden sind. Wichtige Fragen in diesem Zusammenhang sind: Welche Grundaussagen «klassischer) Kapitalismusanalyse haben unter den heutigen technologischen, wirtschaftlichen und (welt)gesellschaftlichen Verhältnissen nach wie vor eine Relevanz? Was hat sich im digitalen Kapitalismus verändert?

Folgt man verschiedenen, im vorigen Teil skizzierten Analysen, lassen sich folgende Grundaussagen zu kapitalistischen Strukturprinzipen nennen, die auch heute eine Gültigkeit bzw. auf dem Hintergrund voranschreitender technologischer und finanzkapitalistischer Entwicklungen eine zunehmende Relevanz haben:

a) Prinzip der Kapitalakkumulation (Profitstreben) auf der Basis von Privateigentum (Grundbesitz, Rohstoffe, Produktionsmittel, Aktien etc.); damit verknüpft: Prinzip des quantitativen Wachstumsdenkens, um sich auf dem kapitalistisch strukturierten (Finanz-)Markt durchzusetzen;

b) Prinzip der Monopolbildung, um dauerhaft durch Kapitalkonzentrationsprozesse eine Vorherrschaft auf dem kapitalistisch strukturierten (Welt-)Markt zu erreichen; damit verknüpft: Globalisierung im Sinne der Erschliessung und Verdichtung weltweit verteilter Entwicklungs-, Produktions-, Distributions- und Absatzorte;

c) Prinzip der Reduktion von Kosten für menschliche Arbeitskräfte, um durch den Einsatz neuer Technologien langfristig Kapital zu sparen; damit verknüpft: Beschleunigung und Flexibilisierung von Arbeitsabläufen; Informatisierung und Immaterialisierung.

d) Prinzip der Ökonomisierung möglichst vieler gesellschaftlicher Bereiche, um der Kapitalakkumulation stets neue Areale zu erschliessen; damit verknüpft: Quantifizierung und Vermessung von Institutionen, «Verbraucher» und "Kunden» - bis in sensible und intimste Bereiche hinein.

Was hat sich im digitalen Kapitalismus gegenüber den bisherigen Formen des Kapitalismus verändert? Die These ist, dass die skizzierten kapitalistischen Strukturprinzipien, die sich in den vergangenen 100 bis 150 Jahren herausgebildet haben, nach wie vor gültig sind und der Kapitalismus durch die Verwertung digitaler Technologien einen gewaltigen Schub erhielt. Dies liegt u. a. darin begründet, dass die skizzierten kapitalistischen Strukturprinzipien eine Affinität zu digitalen Strukturprinzipien haben und dass durch die digitale Technologie (materiell und immateriell) neue gesellschaftliche Areale für die Kapitalakkumulation erschlossen wurden. Es geht um Affinitäten, um die Verknüpfbarkeit beider Strukturprinzipien es werden keine determinierenden Wirkungszusammenhänge unterstellt. Digitale Technologien sind für unterschiedliche Zwecke nutzbar. Dies gilt es immer wieder 
zu reflektieren. An dieser Stelle geht es darum, auf die Verknüpfbarkeit mit kapitalistischen Strukturprinzipien hinzuweisen.

Zunächst zu den digitalen Strukturprinzipien. Zu nennen sind:

a) Prinzip der Binarisierung: Umwandlung analoger Signale in binäre Werte (komplexe Reihungen von 0- und 1-Werten), die nur noch als Rasterpunkte erscheinen (numerische Repräsentation von Daten verschiedenster Art und deren Nutzung, Speicherung, Bearbeitung, Verteilung und Darstellung in einer binär codierten Form); Binarisierung als Voraussetzung für die Algorithmisierung von Prozessen und zu lösenden Problemen.

b) Prinzip der Variabilität und der Simulation als Möglichkeit, in beliebige mediale Artefakte hineinzugehen, sie bitweise neu zu bearbeiten und zu verändern, imaginäre Räume neu zu schaffen. Schrift, Töne, Bilder, Bewegtbilder, Grafiken, Sprache, Geräusche und Musik können elektronisch-maschinell weiterbearbeitet und neu erzeugt werden.

c) Prinzip der Augenblicklichkeit: Die Informationen, Töne, Bilder etc. werden in Lichtgeschwindigkeit transportiert und ermöglichen Interaktivität und weltweite Vernetzung in Echtzeit (Punkt-zu-Punkt-Erreichbarkeit).

d) Prinzip der Miniaturisierung von Einzelbausteinen zu Funktionsblöcken, deren zeitliche Schaltungen sich im Nanosekundenbereich (milliardstel Sekunden) bewegen, sowie Prinzip der Modularisierung, d.h. der beliebigen Austauschbarkeit und Kompilation digitaler Produktionsteile. ${ }^{10}$

Nun zur Affinität zwischen digitalen und kapitalistischen Strukturprinzipien. Was sind Kernpunkte dieser Affinität bzw. Verknüpfbarkeit?

Zunächst lässt sich festhalten, dass die Quantifizierung ein grundlegendes gemeinsames Merkmal ist: Die Messbarkeit von Prozessen, Kostenfaktoren, Tauschwerten, Profitraten (in Verbindung mit einem quantitativen Wachstumsdenken) korrespondiert auf das Beste mit der numerischen Repräsentation von Daten verschiedenster Art. Aktuell sind z. B. Big Data-Projekte gefragt, die angesichts riesiger Datenmengen auf der Basis algorithmischer Verfahren eine gezielte, rationale und effiziente Filterung und Aufbereitung für jeweils spezifische Verwertungszwecke (nicht nur für Produktwerbung) ermöglichen (vgl. Betz/Kübler 2013, 237 f.). In Verknüpfung mit dem digitalen Strukturprinzip der Augenblicklichkeit können in diesen quantifizierenden Prozessen - gemäss dem Motto «Zeit ist Geld!» - z. B. im Hochfrequenzhandel an den Börsen bei Transaktionen riesige Kapitalgewinne in kürzester Zeit erzielt werden. Diese Form des Finanzkapitalismus entkoppelt Wertschöpfungsprozesse völlig von konkreten Gegenständen und menschlicher Arbeit und simuliert finanzielle Welten, die keine Entsprechung in der so genannten Realökonomie haben (Entgrenzung von Kapitalbewegungen).

10 Zu den genannten digitalen Strukturprinzipien vgl. u. a. Niesyto (2002) und Presse- und Informationsamt der Bundesregierung, Hrsg. $(2012,489)$. 
Generell ermöglicht die digitale Vernetzung und Interaktivität die ständige und flexible Verfügbarkeit von Menschen und Maschinen. Insbesondere räumliche und zeitliche Flexibilitätsanforderungen an die Arbeitskräfte lassen sich durch den Einsatz digitaler Technologien gezielt umsetzen. Aus diversen Arbeitsfeldern ist bekannt, dass die ständige Erreichbarkeit, Beschleunigung und Verdichtung von Arbeitsabläufen aus Sicht der betroffenen Mitarbeiter/innen im Falle fehlender Regulierungen (zeitliche Grenzziehungen) zu permanenter Überlastung, Überforderung und Stress führen können. Die flexible, weltweite Verfügbarkeit von Menschen und Maschinen ist wesentlich, um als 'global players Kapitalakkumulation in Richtung Kapitalkonzentration und Monopolbildung vorantreiben zu können (Suche nach den effizientesten Entwicklungs-, Produktions-, Distributionsorten und deren globale Vernetzung).

Die digitalen Strukturprinzipien der Variabilität und der Simulation passen sehr gut zu kapitalistischen Strukturprinzipien, die auf permanente Anpassungsfähigkeit, Effizienz und ökonomische Verwertbarkeit abzielen. Das «Internet der Dinge` ist ein Bereich, in welchem aktuell sehr deutlich wird, wie das Ineinander von realen, körperlich-physikalischen und immateriellen Datenwelten (auch Simulationen) immer fliessender und intensiver wird. Die Vermessung nahezu aller Lebenswelten und des eigenen Körpers (Self-Tracking etc.) offerieren die Optimierung des Alltags und des Selbst, haben allerdings einen harten kapitalistischen Kern: die Erschliessung neuer Absatzmärkte, die Ökonomisierung immer weiterer Lebensbereiche, die Kontrolle und Überwachung des Körpers. ${ }^{11}$

Die Miniaturisierung und Modularisierung digitaler Bausteine sind wichtige technologische Voraussetzungen, um den Arbeits- und Materialaufwand zu reduzieren, die Fertigungstechnik und Logistik bei der Maschine-zu-Maschine-Kommunikation zu informatisieren (Industrie 4.0) und um die Mensch-Computer-Schnittstelle in Richtung Mensch 2.0/human enhancement zu forcieren. Ursprünglich vor allem im Bereich assistiver Computertechnologie (zur Unterstützung von Menschen mit Behinderungen) entwickelt, zeichnen sich hier zahlreiche neue Anwendungsfelder ab. Während in der Vergangenheit Medientechnologien vor allem Formen der medialen Extension ermöglichten (in die Ferne zu gelangen), erweitern digitale Technologien diese Möglichkeiten nun ins Körperinnere hinein (dies soll als mediale Inkorporation bezeichnet werden). Es ist zu vermuten, dass vor allem die Bioinformatik künftig von diversen Wirtschaftsunternehmen heftig umworben wird, um durch den Einsatz entsprechender Technologien neue Märkte zu erschliessen. Damit verbunden sind grundlegende anthropologische, ethische und soziale Fragestellungen von Mensch- und Subjekt-Sein in digitalisierten und ökonomisierten

11 In Verbindung mit der Erfassung von eigenen Körperdaten wird z. B. prognostiziert, dass künftig von Krankenkassen Versicherungstarife (auch) unter Bezug auf eigene Fitness-Anstrengungen etc. zugeordnet werden. 
Gesellschaften (Stichworte: Abschied vom Autonomie-Gedanken des Subjekts, neuartige totalitäre Überwachungs- und Herrschaftsformen, Übergang zu einem «relationalen Selbst»; vgl. Zöllner 2016).

Die skizzierten Entwicklungstrends haben das Potenzial, Mensch-Sein, Alltag und Arbeit sowie das Zusammenleben in der Gesellschaft grundlegend zu verändern. Kritisch zu bewerten sind insbesondere technologiegetriebene Zukunftsmodelle vom gesellschaftlichen Zusammenleben, die die Vielschichtigkeit des Menschseins letztlich der Präzision algorithmischer Berechnungen und Eindeutigkeiten unterordnen. Es geht um grundlegende Kriterien und Werte, um Bilder vom Menschen und der Gesellschaft und die Frage, ob weiterhin eine Pluralität von Konzepten und Vorstellungen existiert oder ob Verengungen auf bestimmte Leitbilder um sich greifen. Die Affinität zwischen kapitalistischen und digitalen Strukturprinzipien, das Dominanzstreben global agierender Konzerne, die Kopplung dieser Strukturen mit individualistischen Konzepten der Selbstoptimierung drohen gesellschaftliche Kräfte zu marginalisieren, die gegenüber diesem Mainstream andere Vorstellungen von Lebensgestaltung, von Selbstbestimmung, von Gemeinschaftlichkeit und gesellschaftlichen Zukunftsentwicklungen befördern möchten. Gleichzeitig ist festzuhalten, dass digitale Technologien in zahlreichen Bereichen für aufklärerische, demokratische und partizipative Zwecke genutzt werden. Wir haben es mit einer paradoxen Situation zu tun: Einerseits beweist der Kapitalismus seine Selbsterneuerungskraft dadurch, dass er die systematische Verwertung digitaler Daten ins Zentrum seiner Kapitalakkumulationen rückt - und es offensichtlich dabei schafft, entsprechende Daten-Enteignungsprozesse grossen Teilen der Bevölkerung als unproblematische Begleiterscheinung und sogar als persönlichen Vorteil zu verkaufen (nach dem Motto: «lch habe nichts zu verbergen, bekomme dafür gezielt Informationen und Angebote und kann am Leben vieler Menschen teilnehmen»). Andererseits verbinden sich mit der aktiven Nutzung digitaler Technologien Hoffnungen wie sharing economy, Dezentralisierung, Kooperation, offene Zugänge statt Eigentumsprinzip, Demokratisierung der Fabrikation, Stärkung sozial-ökologischer Wirtschaftsformen etc. (vgl. u. a. Habermann 2015). Diese Paradoxien werden in den Subjekten ausgetragen und sind von ihnen zu bewältigen. Sie zu benennen und sich mit ihnen auseinandersetzen, ist auch eine gesellschaftliche Aufgabe - für Bildung, Wissenschaft und andere Bereiche. ${ }^{12}$

12 vgl. in diesem Kontext z. B. die Plattform «Understanding Digital Capitalism». Zugriff 27.10.2016 http://dasfilter.com/gesellschaft/introducing-understanding-digital-capitalism-in-was-fuer-zeitenleben-wir 


\section{Zur gesellschaftlichen Verantwortung der Medienpädagogik}

Der vorliegende Beitrag erhebt nicht den Anspruch einer eingehenden Analyse. Die Medienpädagogik steht gegenwärtig noch im Anfangsstadium einer vertieften Auseinandersetzung mit strukturellen Fragen eines digitalen Kapitalismus. Diese Auseinandersetzung ist notwendig, um die künftigen Entwicklungspfade der Medienpädagogik weiter zu klären: entweder immer mehr zu einem Ausbildungs- und Reparaturbetrieb des digitalen Kapitalismus für die berufsbezogene Anwendung digitaler Technologien und deren «Risiken und Nebenwirkungen` zu werden oder sich als kritisch-reflexive Begleitung von medienbezogenen Bildungs- und Lernprozessen zu verstehen, die Formen einer aktiv-produktiven Mediennutzung und -gestaltung mit einer kritisch-reflexiven Aneignung von gesellschaftlichem und digital-medialem Strukturwissen verknüpft. Mein Anliegen ist: Die Medienpädagogik sollte sich verstärkt mit strukturellen Fragen der Digitalisierung in Verbindung von Medien- und Gesellschaftskritik befassen. Dafür ist eine Auseinandersetzung mit vorhandenen kritischen Analysen aus der Wirtschaftswissenschaft und politischen Ökonomie, der Kultur- und Techniksoziologie, der Sozialwissenschaften, der Medien- und Kommunikationswissenschaft, der Informatik und weiteren Bereichen sinnvoll.

Strukturprinzipien digitaler Medien sind zwar anschlussfähig für kapitalistische Strukturprinzipien. Digitale Medien enthalten aber zugleich sehr wichtige Potentiale für die Reflexion und Transformation von Selbst- und Weltverhältnissen und damit für Bildung und Medienbildung. Dies ist der hauptsächliche Ansatzpunkt für die Medienpädagogik. Hierin zu integrieren sind Themen der Medien- und Gesellschaftskritik, anknüpfend an den Wahrnehmungs- und Erfahrungswelten von Kindern, Jugendlichen und Erwachsenen. Mit Blick auf die skizzierten Enteignungsprozesse stellt sich die Frage, durch welche Aktivitäten derartige Prozesse und Mechanismen hinterfragt und durchbrochen werden können und wie die Medienpädagogik Räume für eine Medienbildung eröffnen kann, die Persönlichkeitsbildung und gesellschaftliche Teilhabe in verschiedenen Feldern fördert. Die Janusköpfigkeit digitaler Technologien spiegelt sich in Paradoxien des Umgangs mit ihnen. Dies ist ein wichtiger Ansatzpunkt für Medienbildung: Orientierungs- und Bewältigungsprozesse zu begleiten, Ambivalenzen und Paradoxien im Umgang mit digitalen Medien zu thematisieren und gemeinsam nach Möglichkeiten und Wegen für einen selbstbestimmten und souveränen Umgang mit digitalen Medien zu suchen.

Dazu gehören sowohl die aktiv-produktive und die kritische Auseinandersetzung mit der angebotenen Software und ihrer jeweiligen Datenbasis, die Reflexion von medienästhetischen Mustern (in Verbindung mit ihren symbolischen Bedeutungen), als auch die Zusammenarbeit mit anderen Akteuren, um Anregungen zu erhalten und (idealiter) Ideen z. B. für Alternativen zu marktgängigen Angeboten auf 
den Weg zu bringen. Gerade was die Auseinandersetzung mit informatischen und algorithmischen Fragen und dem damit verbundenen Strukturwissen betrifft, gibt es in der Medienpädagogik einen Nachholbedarf. ${ }^{13}$ Der Einschätzung von Stefan Iske und Dan Verständig ist zuzustimmen:

Die medienpädagogische Kooperation mit weiteren gesellschaftlichen Akteuren und Organisationen zur Gestaltung der Strukturen des Internet wird zu einer zentralen und dauerhaften Aufgabe der Medienpädagogik, so wie es im Rahmen der Initiative Keine Bildung ohne Medien bereits initiiert worden ist. Andernfalls werden vor allem wirtschaftliche und politische Akteure die grundlegenden Infrastrukturen schaffen, auf die Medienpädagogik im Sinne eines «Reparaturbetriebs» lediglich reagieren kann. (Iske/Verständig 2015)

Deshalb brauchen wir eine Medienpädagogik, die technologisch-informatische, ökonomische, kulturelle und soziale, ethische und ästhetische Fragen thematisiert, die das Medienhandeln der Menschen in Zusammenhang mit gesellschaftlichen Medienentwicklungen erforscht und analysiert und die Kindern, Jugendlichen und Erwachsenen in Bildungskontexten Handlungsräume mit Medien jenseits kommerziell-kapitalistischer Vereinnahmungen eröffnet (vgl. Witzel 2012, 89 f.). Dies schliesst auch eine verstärkte Kooperation mit anderen Bildungsbereichen ein wie z. B. der informatischen Bildung (vgl. Fussnote 13), der ökonomischen Bildung (vgl. Hofhues/Schiefner-Rohs 2012) oder der Verbraucherbildung..$^{14}$ Vor allem bedarf es erheblich grösserer Anstrengungen, um pädagogische Fachkräfte in allen pädagogischen Handlungsfeldern für eine medienpädagogische Arbeit angemessen zu qualifizieren (Stichwort: medienpädagogische Grundbildung; vgl. die Forderungen der Initiative «Keine Bildung ohne Medien! - KBoM» und die Analysen und Vorschläge im Sammelband von Imort/Niesyto 2014). ${ }^{15}$

Unabhängig von diesen Anstrengungen existieren weiterhin gesellschaftlichmediale Strukturen, die die sozialen Lebenswelten der Menschen durchdringen und überformen. Diese Strukturen sind zu analysieren, zu kritisieren und - auch im Rahmen eines professions- und bildungspolitischen Engagements - zu verändern. Die Möglichkeiten der Medienpädagogik sind dafür begrenzt, aber sie sollte nach

13 vgl. in diesem Zusammenhang einzelne Beiträge in diesem Band zur Relevanz informatischer Bildung, das Themenheft Nr. 25 der Online-Zeitschrift MedienPädagogik zum Thema «Medienbildung und informatische Bildung - quo vadis?» (http://www.medienpaed.com/issue/view/31) sowie die «Dagstuhl-Erklärung» (2016) zur Bildung in der digitalen vernetzten Welt (https://www.gi.de/aktuelles/meldungen/detailansicht/article/dagstuhl-erklaerung-bildung-in-der-digitalen-vernetzten-welt. html).

14 vgl. hierzu die interdisziplinäre Tagung des JFF München über «Medien, Konsum, Kultur» (2015), auf der der aktuelle Stand der wissenschaftlichen Auseinandersetzung zu den Zusammenhängen zwischen Medienhandeln und Konsumhandeln diskutiert und Vorschläge für die medienpädagogische Praxis entwickelt wurden. Kurzbericht: http://www.jff.de/jff/themen/news/artikel/art/idt15-abstractsder-hauptvortraege-online-kalenderdatei-eintragung-in-fibs/

15 KBoM: http://www.keine-bildung-ohne-medien.de/pages/grundbildung-medien/ 
Kräften ihre Stimme zusammen mit anderen Akteuren erheben (vgl. die Initiative KBoM und andere Aktivitäten). Ein Denken, das vom Primat wirtschaftlicher Interessen und stetiger ökonomischer Wachstumsszenarien ausgeht und hierunter das sog. «Humankapital» zu- und unterordnet, konfligiert grundsätzlich mit einem Verständnis von Bildung und Medienbildung, welches die Menschen mit ihren unterschiedlichen Lebensbedürfnissen in den Mittelpunkt stellt. Medienpädagogik kommt nicht umhin, sich in der Klärung und (Weiter-)Entwicklung ihrer Aufgabenstellungen mit diesen Fragen zu befassen und sich im Kontext gesamtgesellschaftlicher Entwicklungen zu verorten. Dies bedeutet auch, Medienbildung wieder verstärkt als politisch-kulturelle Medienbildung zu begreifen. ${ }^{16}$ Thomas Krüger, Präsident der Bundeszentrale für politische Bildung, äusserte sich hierzu deutlich:

Die Herausforderung wäre, der Frage nachzugehen, ob die Medienpädagogik nochmal als eine «kritische Gesellschaftsanalyse», wie es Baacke gefordert hatte, zu konstituieren sei, ohne damit die vergangenen drei bis vier Jahrzehnte theoretischer Diskussion zu negieren, sondern produktiv in eine Neubestimmung zu integrieren (Krüger 2014).

Medienpädagogik als kritische Medien- und Gesellschaftsanalyse und zugleich als subjektorientierte Handlungswissenschaft und pädagogisches Arbeitsfeld zu entwickeln, ist kein Widerspruch. Wir benötigen beides: Die konsequente Orientierung an den Menschen, ihren Bedürfnissen, Interessen und Lebensformen, aber auch die Untersuchung struktureller (und sozialisationsrelevanter) gesellschaftlichmedialer Muster, die Wahrnehmung, Denken und Handeln der Menschen beeinflussen. Besonders wichtig ist das Offensein für Widerständigkeiten und die Unterstützung der Artikulation von eigensinnigen Vorstellungen, um das Selbstbewusstsein zu stärken und Räume für ein Denken in Alternativen zu befördern. Die Widerständigkeit von Subjekten gesellschaftlichen Verhältnissen gegenüber, die sie zu vereinnahmen suchen, war schon immer eine wichtige Intention einer kritischreflexiven Erziehungswissenschaft (vgl. Kammerl 2016). Diese Intention grenzt sich von utilitaristischen Auffassungen ab, die die Zurichtung von Menschen im Sinne von 〈Verwendungstauglichkeit» intendieren, und eröffnet eine Perspektive, über den Status quo hinauszudenken. Für den Pädagogen Heinz-Joachim Heydorn war Bildung in dieser Perspektive eine «entbundene Selbsttätigkeit», eine «Selbstverfügung», in welcher der Mensch als Subjekt in seine eigene Geschichte eintritt. Bildung sei die Befähigung des Menschen zur gesellschaftlichen Arbeit und zum politischen Handeln, aber auch zur ästhetischen Erfahrung, in welcher der Mensch sich selbst vergegenwärtige. Heydorn ging von der Dialektik von institutionalisierter

16 Dieser Intention liegt ein Politikverständnis zugrunde, welches sich nicht auf Formen der parlamentarischen Interessenvertretung reduziert, sondern insgesamt die demokratische Teilhabe von Menschen am Gemeinwesen und in der Gesellschaft im Blick hat und sie darin unterstützen möchte, (digitale) Medien aktiv für Partizipation und öffentliches Handeln zu nutzen und hierüber Selbstwirksamkeit zu erfahren. 
Bildung und menschlicher Selbstbildung aus und betonte die Notwendigkeit von Distanzierungsmöglichkeiten zur Ausbildung von Widerstandspotentialen gegenüber gesellschaftlichen Herrschaftsstrukturen (vgl. Sünker 2001, 163 f.). In dieser Perspektive lässt sich Bildung allgemein als selbstreflexiver Lern- und Orientierungsprozess fassen, der nicht - wie Winfried Marotzki und Benjamin Jörissen betonen - auf ein bestimmtes Ergebnis oder einen bestimmten Zustand abzielt, sondern darauf, "vorhandene Strukturen und Muster der Weltaufordnung durch komplexere Sichtweisen auf Welt und Selbst» zu ersetzen (Marotzki/Jörissen 2010, 19). Dies impliziere die Fähigkeit zur Distanzierung gegenüber vorhandenen Strukturen und Mustern der Weltaufordnung (ebd., 22). ${ }^{17}$

In einer historischen Epoche, in welcher die Digitalisierung nahezu alle gesellschaftlichen Bereiche nachhaltig verändert, wird Medienbildung zu einer Kernaufgabe von Bildung und damit auch zur Hinterfragung vorhandener Strukturmuster eines digitalen Kapitalismus. In einer Situation, in der global agierende Medienkonzerne, Wirtschaftsunternehmen und andere Einrichtungen und Organisationen immer extensiver persönliche Daten ausspähen und für ihre jeweiligen Zwecke verwenden, ist es wichtig, verstärkt Datenkritik zu entwickeln (vgl. Dander 2014) und an dem Recht auf informationelle Selbstbestimmung festzuhalten. ${ }^{18}$ Es ist wichtig, angesichts einer allumfassenden Quantifizierung und Vermessung von Leben und Gesellschaft Fragen nach Sinn und Bedeutung jenseits dieser quantifizierenden Prozessstrukturen zu stellen. Hierfür sollte eine kritisch-reflexive und politischkulturelle Medienbildung Gelegenheiten eröffnen und Kinder, Jugendliche und Erwachsene darin unterstützen, digitale Medien selbstbestimmt und kompetent zu nutzen (vgl. hierzu auch das Konzept «Digital Citizenship», Moser 2016).

Eine handlungsorientierte Medienkritik gehört zu den wesentlichen Aufgaben der Medienpädagogik. Eine solche Medienkritik geht von den medialen Alltagserfahrungen der Menschen aus und befördert eine altersangemessene Auseinandersetzung mit konkreten Handlungsmöglichkeiten. Entsprechende Angebote, Konzepte und Materialien für schulische und ausserschulische Bildungsorte sind verstärkt zu entwickeln. Gleichzeitig gilt es, den Druck der Zivilgesellschaft in Richtung Politik und (Internet-)Wirtschaft zu verstärken und erheblich bessere strukturelle Rahmenbedingungen für informationelle Selbstbestimmung einzufordern: «Digitale Selbstbestimmung kann nicht individualisiert werden, sondern ist auf einen (zivil-)

17 Dieses Verständnis schliesst nicht aus, dass z. B. im Kontext formaler Bildungsprozesse in Schulen auch Standards definiert werden, gerade im Hinblick auf Mindeststandards der Medienkompetenz. Dies ist eine wichtige Frage, um einen Beitrag gegen bestehende Bildungsungerechtigkeit zu leisten. Eine andere Frage ist die notwendige Kritik an output-orientierten Kompetenz-Katalogen, die Bildungsprozesse in einem Korsett aus engmaschig gestrickten Teilkompetenzen ersticken.

18 vgl. in diesem Kontext auch Das Digital-Manifest: www.spektrum.de/news/wie-algorithmen-undbig-data-unsere-zukunft-bestimmen/1375933 ("Digitale Demokratie statt Datendiktatur”). Nach Verfassen des vorliegenden Beitrags erschien von einer Autoren/innengruppe ein wichtiges medienpädagogisches Diskussionspapier zu Big Data und Data Analytics, welches prägnant gesellschaftliche Problemfelder und Aufgaben der Medienpädagogik darstellt (vgl. Aßmann et al. 2016). 
gesellschaftlich verankerten Handlungsrahmen angewiesen ist, der diese ermöglicht» (Aßmann et al. 2016).

\section{Literaturverzeichnis}

Aßmann, Sandra, Niels Brüggen, Valentin Dander, Harald Gapski, Gerda Sieben, Angela Tillmann, und Isabel Zorn. 2016. «Digitale Datenerhebung und -verwertung als Herausforderung für Medienbildung und Gesellschaft. Ein medienpädagogisches Diskussionspapier zu Big Data und Data Analytics». In Kommunikationskulturen in digitalen Welten, hrsg. v. Marion Brüggemann, Thomas Knaus und Dorothee Meister. München: kopaed.

Autenrieth, Daniel. 2016. «Meinungsbildungsprozesse unter dem Einfluss digitaler Medien und deren Gefahrenpotenziale für demokratische Systeme». In medien impulse, Ausgabe 2/2016. http://www.medienimpulse.at/articles/view/922.

Baacke, Dieter. 1997. Medienpädagogik. Tübingen: Niemeyer.

Betz, Joachim, Kübler, Hans-Dieter. 2013. Internet Governance. Wer regiert wie das Internet? Wiesbaden: Springer VS.

Biermann, Ralf. 2009. «Die Bedeutung des Habitus-Konzepts für die Erforschung soziokultureller Unterschiede im Bereich der Medienpädagogik». Hrsg. v. Horst Niesyto, Dorothee Meister, und Heinz Moser. MedienPädagogik: Zeitschrift für Theorie und Praxis der Medienbildung, Nr. 17 Medien und soziokulturelle Unterschiede (August): 1-18. doi:10.21240/mpaed/17/2009.08.14.X.

Böhnisch, Lothar, Karl Lenz, und Wolfgang Schröer. 2009. Sozialisation und Bewältigung. Eine Einführung in die Sozialisationstheorie der zweiten Moderne. Weinheim/München: Juventa.

Böhnisch, Lothar, und Wolfgang Schröer. 2001. Pädagogik und Arbeitsgesellschaft. Historische Grundlagen und theoretische Ansätze für eine sozialpolitisch reflexive Pädagogik. Weinheim und München: Juventa Verlag.

Boltanski, Luc, und Ėve Chiapello. 2003. Der neue Geist des Kapitalismus. Konstanz: UVK.

Bos, Wilfried, Birgit Eickelmann, Julia Gerick, Frank Goldhammer, Heike Schaumburg, Knut Schwippert, Martin Senkbeil, Renate Schulz-Zander, und Heike Wendt, Hrsg. 2014. ICILS 2013. Computer- und informationsbezogene Kompetenzen von Schülerinnen und Schülern in der 8. Jahrgangsstufe im internationalen Vergleich. Münster: Waxmann. https:// www.waxmann.com/fileadmin/media/zusatztexte/ICILS_2013_Berichtsband.pdf

Damberger, Thomas. 2016. «Zur Information: Der blinde Fleck im Transhumanismus». In FIfF-Kommunikation. Zeitschrift für Informatik und Gesellschaft (Heft 2/2016). Bremen: FIfF, 32-36.

Dander, Valentin. 2014. Von der ,Macht der Daten' zur, Gemachtheit von Daten'. Praktische Datenkritik als Gegenstand der Medienpädagogik. http://www.medialekontrolle.de/ wp-content/uploads/2014/09/Dander-Valentin-2014-03-01.pdf

Dörre, Klaus, Stephan Lessenich, und Hartmut Rosa. 2010. Soziologie - Kapitalismus - Kritik: eine Debatte. Frankfurt am Main: Suhrkamp.

Dolata, Ulrich. 2014. Märkte und Macht der Internetkonzerne. http://www.uni-stuttgart.de/ soz/oi/publikationen/soi_2014_4_Dolata_Maerkte_und_Macht_der_Internetkonzerne. pdf.

Ehmer, Herrmann K. 1971. Visuelle Kommunikation. Beiträge zur Kritik der Bewusstseinsindustrie. Köln: DuMont. 
Fuchs, Christian. 2014. Digital Labour and Karl Marx. New York: Routledge.

Ganguin, Sonja, und Uwe Sander. 2008. «Identitätskonstruktionen in digitalen Welten». In Handbuch Medienpädagogik, hrsg. von Uwe Sander, Friederike Gross, und Kai-Uwe Hugger, 422-427. Wiesbaden: VS Verlag für Sozialwissenschaften.

Gapski, Harald, Hrsg. 2015. Big Data und Medienbildung. Zwischen Kontrollverlust, Selbstverteidigung und Souveränität in der digitalen Welt. Schriftenreihe zur digitalen Gesellschaft NRW, Bd. 3. München: kopaed.

Glotz, Peter. 1999. Die beschleunigte Gesellschaft. Kulturkämpfe im digitalen Kapitalismus. München: Kindler.

Göttlich, Udo. 2015. «Materialität, Ökonomie und Markt». In Cultural Studies und Medienanalyse, hrsg. von Andreas Hepp, Friedrich Krotz, Swantje Lingenberg, und Jeffrey Wimmer, 31-38. Wiesbaden: Springer VS.

Habermann, Friederike. 2015. "Commonsbasierte Zukunft. Wie ein altes Konzept eine bessere Welt ermöglicht». In Aus Politik und Zeitgeschichte, 65. Jahrgang, 35-37 (2015): 46-52.

Herrmann, Ulrike. 2015. "Vom Anfang und Ende des Kapitalismus». In Aus Politik und Zeitgeschichte, 65. Jahrgang, 35-37 (2015): 3-9.

Habermas, Jürgen. 1987. Theorie kommunikativen Handelns, 4. Auflage. Frankfurt/Main: Suhrkamp.

Haug, Wolfgang Fritz. 2012. Hightech-Kapitalismus in der grossen Krise. Hamburg: Argument.

Hipfl, Brigitte, und Katharina Ernst, Hrsg. 2004. Der Beitrag der Cultural Studies für die Medienpädagogik. MedienPädagogik Themenheft 8. doi:10.21240/mpaed/08.X.

Hofhues, Sandra, und Mandy Schiefner-Rohs. 2012. "Crossing Boundaries. Handeln in Medienprojekten zwischen Medienbildung und ökonomischer Bildung». In medien+erziehung, 56. Jhg, Nr. 6 (2012): 93-102.

Hofstetter, Yvonne. 2014. Sie wissen alles. Wie intelligente Maschinen in unser Leben eindringen und warum wir für unsere Freiheit kämpfen müssen. München: C. Bertelsmann.

Horkheimer, Max, Theodor W. Adorno. (1944) 1960. Dialektik der Aufklärung. Frankfurt/ Main: S. Fischer.

Hurrelmann, Klaus. 1995. Einführung in die Sozialisationstheorie. Über den Zusammenhang von Sozialstruktur und Persönlichkeit. Weinheim/Basel: Beltz.

Imort, Peter, Niesyto, Horst. 2014. Grundbildung Medien in pädagogischen Studiengängen. München: kopaed.

Iske, Stefan. 2014. «Medienbildung» In Medienpädagogik - ein Überblick, hrsg. v. Friedricke von Gross, Dorothee M. Meister, und Uwe Sander, 247-272. Weinheim: Beltz Juventa.

Iske, Stefan, und Dan Verständig. 2014. «Medienpädagogik und die Digitale Gesellschaft im Spannungsfeld von Regulierung und Teilhabe». In medienimpulse, Nr. 4 (2014). http:// www.medienimpulse.at/articles/view/751.

Iske, Stefan, Alexandra Klein, Nadia Kutscher, und Hans-Uwe Otto. 2007. «Virtuelle Ungleichheit und informelle Bildung. Eine empirische Analyse der Internetnutzung Jugendlicher und ihre Bedeutung für Bildung und gesellschaftliche Teilhabe». In: Kompetenzzentrum Informelle Bildung (Hrsg.): Grenzenlose Cyberwelt? Zum Verhältnis von digitaler Ungleichheit und neuen Bildungszugängen für Jugendliche, 65-91. Wiesbaden: VS Verlag für Sozialwissenschaften. 
Jörissen, Benjamin. 2015. «Transgressive Artikulation: Ästhetik und Medialität aus Perspektive der strukturalen Medienbildung». In Medienkultur und Bildung, hrsg. v. Malte Hagener, und Vinzenz Hediger, 49-64. Frankfurt am Main: Campus.

Jörissen, Benjamin, und Winfried Marotzki. 2009. Strukturale Medienbildung - Eine Einführung. Bad Heilbrunn: Klinkhardt.

Kammerl, Rudolf. 2016. «Medien-Bildung wozu? Hat Medien-Bildung Zwecke und wenn ja, wer legt diese warum fest?» In Medien - Wissen - Bildung, hrsg. von Theo Hug, Tanja Kohn, und Petra Missomelius, 139-149. Innsbruck: University Press.

Kommer, Sven. 2010. Kompetenter Medienumgang? Eine qualitative Untersuchung zum medialen Habitus und zur Medienkompetenz von SchülerInnen und Lehramtsstudierenden. Leverkusen: Budrich UniPress Ltd.

Krotz, Friedrich. 2015. «Der Wandel jugendspezifischer Kommunikationskulturen im Prozess der Mediatisierung». Plenumsvortrag auf dem 32. Forum Kommunikationskultur der GMK. http://www.gmk-net.de/fileadmin/pdf/Praesentationen/forum2015_krotz_CClizenz.pdf (Folien).

Krotz, Friedrich. 2014. «Einleitung: Projektübergreifende Konzepte und theoretische Bezüge der Untersuchung mediatisierter Welten». In Die Mediatisierung sozialer Welten. Synergien empirischer Forschung, hrsg. von Friedrich Krotz, Cathrin Despotovic, und Merle-Marie Kruse, 7-32. Wiesbaden: Springer VS.

Krotz, Friedrich. 2012. "Kommunikatives Handeln in ökonomisierten und mediatisierten Welten. Ein Essay zur Einführung». In medien+erziehung, 56. Jhg, Nr. 6 (2012): 7-16.

Krüger, Thomas. 2014. Zwischen Filterblase und snapchat. Politische Partizipation im digitalen Wandel und die neuen/alten Aufgaben der Medienpädagogik. http://www.bpb. de/presse/196663/impulsrefererat-von-thomas-krueger-beim-31-gmk-forum-kommunikationskultur-doing-politics-politisch-agieren-in-der-digitalen-gesellschaft-am-21-11-2014-in-berlin.

Kübler, Hans-Dieter. 2015. Rezension zum Handbuch «Cultural Studies und Medienanalyse», hrsg. von Andreas Hepp, Friedrich Krotz, Swantje Lingenberg, Jeffrey Wimmer. Wiesbaden: Springer VS. http://www.rkm-journal.de/archives/18402.

Kübler, Hans-Dieter. 2006. «Zurück zum «kritischen Rezipienten`? Aufgaben und Grenzen pädagogischer Medienkritik». In Medienkritik heute. Grundlagen, Beispiele, Praxisfelder, hrsg. v. Horst Niesyto, Matthias Rath, und Hubert Sowa, Schriftenreihe medienpädagogik interdisziplinär, Band 5, 17-52. München: kopaed.

Kübler, Hans-Dieter. 2000. Mediale Kommunikation. Tübingen: Niemeyer.

Lamla, Jörn. 2010. «Kultureller Kapitalismus im Web 2.0. Zur Analyse von Segmentations-, Intersektions- und Aushandlungsprozessen in den sozialen Welten des Internets». In Zeitschrift für Qualitative Forschung (ZQF), Jg. 11, H. 1, 11-36. http://www.budrich-journals.de/index.php/zqf/article/view/5102.

Marx, Karl (1867): Das Kapital. Band I. Hamburg: Verlag Otto Meissner.

Müller, Renate, Stefanie Rhein, und Patrick Glogner. 2004. «Das Konzept musikalischer und medialer Selbstsozialisation widersprüchlich, trivial, überflüssig?» In Jugendsoziologische Sozialisationstheorie. Impulse für die Jugendforschung, hrsg. v. Dagmar Hoffmann, und Hans Merkens, 237-252. Weinheim und München: Juventa.

Moser, Heinz. 2016. «Digital Citizenship als Leitlinie der Medienbildung». In Medien - Wissen - Bildung, hrsg. von Theo Hug, Tanja Kohn, und Petra Missomelius, 211-229. Innsbruck: University Press. 
Niesyto, Horst. 2012. «Bildungsprozesse unter den Bedingungen medialer Beschleunigung». In Raum, Zeit, Medienbildung. Untersuchungen zu medialen Veränderungen unseres Verhältnisses zu Raum und Zeit, hrsg. von Gerhard Chr. Bukow, Johannes Fromme, und Benjamin Jörissen, Reihe Medienbildung und Gesellschaft, Band 23, 47-66. Wiesbaden: VS Verlag für Sozialwissenschaften.

Niesyto, Horst, Dorothee M. Meister, und Heinz Moser, Hrsg. 2009. Medien und soziokulturelle Unterschiede. MedienPädagogik Themenheft Nr. 17. doi:10.21240/mpaed/17.X.

Niesyto, Horst. 2008. «Medienkritik». In Handbuch Medienpädagogik, hrsg. v. Uwe Sander, Friederike von Gross, und Kai-Uwe Hugger, 129-135. Wiesbaden: VS Verlag für Sozialwissenschaften.

Niesyto, Horst. 2007. «Kritische Anmerkungen zu Theorien der Mediennutzung und Mediensozialisation». In: Mediensozialisationstheorien. Neue Modelle und Ansätze in der Diskussion, hrsg. von Dagmar Hoffmann, und Lothar Mikos, 47-54. Wiesbaden: VS Verlag für Sozialwissenschaften.

Niesyto, Horst. 2002. «Medien und Wirklichkeitserfahrung - symbolische Formen und soziale Welt». In Wechselbeziehungen Medien, Wirklichkeit, Erfahrung, hrsg. v. Lothar Mikos, und Norbert Neumann, 29-54. Berlin: Vistas. http://www.support-netz.de/fileadmin/user_upload/Medienbildung_MCO/fileadmin/bibliothek/niesyto_wirklichkeit/niesyto_wirklichkeit.pdf.

Niesyto, Horst. 2000. Medienpädagogik und soziokulturelle Unterschiede. Baden-Baden/ Ludwigsburg: Medienpädagogischer Forschungsverbund Südwest.

Niesyto, Horst. 1991. Erfahrungsproduktion mit Medien. Weinheim und München: Juventa. Pariser, Eli. 2012. Filter Bubble. Wie wir im Internet entmündigt werden. München: Hanser.

Paus-Hasebrink, Ingrid, und Christina Ortner. 2007. "Socially Disadvantaged Groups and their Attitudes towards Europe». In Medien Journal, Nr. 2 (2007): 11-21.

Pfadenhauer, Michaela, und Tilo Grenz. 2012. «Anzeichen einer neuen Partizipationskultur? Zur Konsumententolle in mediatisierten Geschäftsmodellen». medien+erziehung, 56. Jhg, Nr. 6 (2012): 17-32.

Piketty, Thomas. 2014. Das Kapital im 21. Jahrhundert. München: C.H. Beck.

Presse- und Informationsamt der Bundesregierung, Hrsg. 2012. Dialog über Deutschlands Zukunft. Ergebnisbericht des Expertendialogs der Bundeskanzlerin 2011/2012. https:// www.bundesregierung.de/Content/Infomaterial/BPA/Bestellservice/2012-09-27-buergerdialog-expertenbericht-langfassung.pdf?_blob=publicationFile\&v=4.

Rifkin, Jeremy. 2014. Die Null-Grenzkosten-Gesellschaft. Das Internet der Dinge, kollaboratives Gemeingut und der Rückzug des Kapitalismus. Frankfurt am Main: Campus.

Rosa, Hartmut. 2009. «Kapitalismus als Dynamisierungsspirale - Soziologie als Gesellschaftskritik». In Soziologie - Kapitalismus - Kritik: eine Debatte, hrsg. von Klaus Dörre, Stephan Lessenich, und Hartmut Rosa, 87-125. Frankfurt am Main: Suhrkamp.

Rosa, Hartmut. 2005. Beschleunigung. Die Veränderung der Zeitstrukturen in der Moderne. Frankfurt am Main: Suhrkamp.

Röll, Franz Josef. 1998. Mythen und Symbole in populären Medien. Der wahrnehmungsorientierte Ansatz in der Medienpädagogik. Frankfurt/Main: Gemeinschaftswerk für Evang. Publizistik.

Schell, Fred. 1993. Aktive Medienarbeit mit Jugendlichen. München: kopaed.

Schiller, Dan. 1999. Digital Capitalism. Cambridge/London: MIT Press. 
Schirrmacher, Frank. 2009. Payback. Warum wir im Informationszeitalter gezwungen sind zu tun, was wir nicht tun wollen, und wie wir die Kontrolle über unser Denken zurückgewinnen. München: Karl Blessing Verlag.

Schorb, Bernd. 1995. Medienalltag und Handeln. Medienpädagogik in Geschichte, Forschung und Praxis. Opladen: Leske + Budrich.

Sennett, Richard. 1998. Der flexible Mensch. Die Kultur des neuen Kapitalismus. Berlin: Berlin Verlag.

Sünker, Heinz. 2001. «Bildung». In Handbuch Sozialarbeit Sozialpädagogik, hrsg. von HansUwe Otto, und Hans Thiersch, 162-168. Neuwied: Luchterhand.

Theunert, Helga, Hrsg. 2010. Medien. Bildung. Soziale Ungleichheit. Differenzen und Ressourcen im Mediengebrauch Jugendlicher. Schriftenreihe Interdisziplinäre Diskurse, Band 5. München: kopaed.

Thomas, Tanja. 2015. «Ideologie, Hegemonie und Diskurs». In Cultural Studies und Medienanalyse, hrsg. von Andreas Hepp, Friedrich Krotz, Swantje Lingenberg, und Jeffrey Wimmer, 67-77. Wiesbaden: Springer VS.

Vogl, Joseph. 2011. Das Gespenst des Kapitals. Zürich: Diaphanes.

Verständig, Dan, und Stefan Iske. 2014. Digitale Teilhabe und digitale Ungleichheiten im Kontext mobiler Internetnutzung. http://blog.hf.uni-koeln.de/medien/files/2011/03/2014_ verst\%C3\%A4ndig-iske_C-U.pdf

Wagner, Ulrike, Hrsg. 2008. Medienhandeln in Hauptschulmilieus. Mediale Interaktion und Produktion als Bildungsressource. München: kopaed.

Witzel, Marc. 2012. «Medienhandeln, digitale Ungleichheit und Distinktion». In medien+erziehung, 56. Jhg, Nr. 6 (2012): 81-92.

Zöllner, Oliver. 2016. «Digitalisierung und Selbstbestimmung». In tv diskurs, 20. Jhg., Nr. 1 (2016): 22-25. 